\title{
LEIBNIZ - IDENTITÄT UND INDIVIDUALITÄT IM DENKEN F. W. J. SCHELLINGS
}

\author{
UDC 111.1+14 LEIBNIZ G.W.+14 Schelling F. W. J.
}

\section{Cristoph Asmuth}

Technische Universität Berlin, Institut für Philosophie, Germany

\begin{abstract}
Abstrakt. Die Interpretation der Leibnizschen Monadenlehre ist bei Schelling transformiert in den eigenen Entwurf einer Identitätstheorie. Dies scheint ein der Philosophie selbst eingeschriebenes Verfahren zu sein: Theoriestücke vergangener Positionen werden zu Bausteinen neuerlicher Theoriebildungen, werden verwandelt und erlangen in verwandelter Gestalt neue Bedeutung. $\mathrm{Zu}$ denken geben muss nur die Tatsache, dass dieser Transformation des Vergangenen oft nur eine unzureichende, bisweilen einseitige, oft kurzsichtige, in jedem Fall aber eine ahistorische Interpretation zugrunde liegt. So interpretiert Schelling in der Tat die Monadenlehre, aber er interpretiert sie von vornherein im Bezugsrahmen der nachkantischen Transzendentalphilosophie: im Horizont der Frage nach dem Ding an sich, der Frage nach der Realität der Außenwelt, der Frage nach der Bewusstseinsimmanenz alles Seienden. Dadurch erscheint die Konzeption von Leibniz insgesamt verkürzt: Die Funktion Gottes als Schöpfer, als Garant einer prästabilierten Harmonie und Stabilisator der Vernunftwahrheiten, als ursprüngliche einfache Substanz (Abschnitt 47) muss in der Transzendentalphilosophie Schellingscher Ausprägung gestrichen werden. Für sie ist kein Ort mehr vorgesehen. An ihre Stelle rückt eine SubjektObjekt-Konzeption, in der die Individualität zwar abstrakt eingelassen ist, deren Basis aber eine Theorie der Selbstverursächlichung bildet, neben der und außerhalb ihrer ein philosophischer Gott nicht bestehen kann. Außerdem fehlen bei Schelling-das sei hier nur grob angedeutet - wesentliche Aspekte: etwa die politischen, sozialen und ethischen.
\end{abstract}

Schlüsselwörter: Leibniz, Schelling, Interpretation, Monadenlehre, Identität, Individualität.

Die Vorstellungen, so Hegel in der Wissenschaft der Logik, erkläre Leibniz in der Monadologie als eine Art Blasen, die im abgeschlossenen Subjekt aufstiegen, Blasen, die deshalb Erscheinungen seien, nicht aber Ding-an-sich und noch weniger Repräsentanten einer Welt-außer-uns: „Die Leibnizische Monade entwickelt aus ihr selbst ihre Vorstellungen; aber sie ist nicht die erzeugende und verbindende Kraft, sondern sie

Received December 11, 2017 / Accepted January 15, 2018

Corresponding author: Cristoph Asmuth

Technische Universität Berlin, Institut für Philosophie, Strasse des 17. Juni 135, D - 10623 Berlin

E-mail: christoph.asmuth@tu-berlin.de 
steigen in ihr als Blasen auf; ..." (Hegel 1978, 247). Die charakteristische Verkürzung, die Leibniz und sein Projekt einer Monadenlehre interpretiert als Einheitslehre im Horizont der nachkantischen Problemlage, ist nicht Hegel allein zu eigen, und sie stammt auch nicht ursprünglich von ihm. Es dürften die Arbeiten des frühen Schelling gewesen sein, angeregt durch die Schriften des Viel-Anregers Friedrich Heinrich Jacobi, ${ }^{1}$ die nach $\mathrm{Kant}^{2}$ - zuerst ein transzendentalphilosophisches Licht auf die Philosophie von Leibniz geworfen haben, weniger die scheinbar verwandte, wenngleich zunächst noch stärker im Schatten Kants stehende Wissenschaftslehre Fichtes. ${ }^{3}$ Schelling jedenfalls war daran gelegen, aufzuzeigen, dass die Vorstellungen in der Leibnizschen Monadenlehre mehr sind als bloße Blasen. Dazu entwickelte Schelling eine transzendentalphilosophische Interpretation der prästabilierten Harmonie. ${ }^{4}$

Für Schelling zählt - zumindest in der ersten Phase seines Philosophierens - Leibniz zu den strahlendsten Göttern seines Philosophenhimmels. Dies allerdings nicht von Anfang an. Es ist meine These, eine These, die ich hier weder belegen noch ausführen möchte, dass sich das philosophische Wirken Schellings in diesen Jahren sehr sprunghaft entwickelte. In der ersten Zeit nach seinem Fortgang aus Jena waren die Schriften stark konzeptionell ausgerichtet: Ihm ging es zunächst um die Entwicklung der Wissenschaftslehre, einem Projekt, über das er sich in jener Zeit noch einig fühlte mit Fichte. Später folgte eine eher eklektizistische Phase, in der sich Schelling auf die Wissenschaften seiner Zeit, speziell die Naturwissenschaften warf, aber auch versuchte, eine historische Anknüpfung seiner - und vermeintlich auch der Fichteschen - Philosophie zu finden. In diese Phase fällt offensichtlich auch seine Auseinandersetzung mit Leibniz, den er - im Kontext seiner kantianisierenden Interpretation Spinozas - gleichfalls unter kantianisierenden Prämissen liest. Es ist eine Zeit, in der Schelling seine philosophischen Götter der Vorzeit meint verteidigen zu müssen gegen eine scheinbar aufgeklärte und superiore Philosophie der Gegenwart. ${ }^{5}$ Die Feinde benennt er und sammelt sie unter den Etiketten Dogmatismus

\footnotetext{
${ }^{1}$ Dabei wirkte Jacobi, zumindest was seine eigenen Interessen betrifft, nicht immer glücklich: Er eröffnete der Generation nach Kant den Weg zu Spinoza und Giordano Bruno, deren philosophisches Wirken er in die Nähe des Pantheismus bzw. Atheismus rückte. Anders seine Wertung Leibniz gegenüber! Schelling konnte sich durch die Schriften Jacobis über Leibniz informieren, etwa aus dem David Hume über den Glauben, oder Idealismus und Realismus. Ein Gespräch (1787), in: (Jacobi 1980, 237-285).

${ }^{2}$ Vgl. hierzu den grundlegenden Aufsatz in systematischer Absicht: Kaehler, Klaus Erich, Von der monadischen zur transzendentalen Subjektivität. Die Bedingtheit in der Unbedingtheit des Subjekts bei Leibniz und Kant, in: (Kaehler 1993, 451-458).

${ }^{3}$ Marco Ivaldo hat unlängst darauf aufmerksam gemacht, wie stark auch für Fichte eine Auseinandersetzung mit Leibniz anzusetzen ist: Marco Ivaldo, Fichte e Leibniz. La comprensione trascendentale della monadologia. Napoli 2000. Allerdings wird man für Fichte eher eine implizite Leibniz-Interpretation ansetzen müssen, da die expliziten Verweise Fichtes auf Leibniz - im Gegensatz zur Leibniz-Interpretation bei Schelling, nur dürftig sind und sich meistens in einer positiven Bewertung ohne nähere Erläuterung erschöpfen. Vgl. dazu (Fichte 1962, 264). Fichte schließt sich Salomon Maimon an, "daß das Leibnizische System, in seiner Vollendung gedacht, nichts anderes sey, als Spinozismus". Und die allgemeine Stellungnahme zu Leibniz: "Denn wohlverstanden (...) hat er recht" (Versuch einer neuen Darstellung der Wissenschaftslehre, GA I, 4, 265), dürfte wohl kaum auf eine inhaltliche Erschließung beim frühen Fichte hindeuten. Vgl. ebenfalls, ders., Das Thema der prästabilierten Harmonie in der Wissenschaftslehre. Untersuchung zur Leibniz-Rezeption J. G. Fichtes, in: VII. Internationaler Leibniz-Kongreß Berlin, 10.-14.9.2001. Nihil sine ratione. Mensch, Natur und Technik im Wirken von G. W. Leibniz. (Hg.) Poser, Hans - Asmuth, Christoph - Goldenbaum, Ursula - Li, Wenchao. 3 Bde. Berlin 2001, Bd. 2, 549-556.

${ }^{4}$ Vgl. dazu: (Hartmut 2000, 127-146).

${ }^{5}$ Schelling, Ideen zu einer Philosophie der Natur als Einleitung in das Studium dieser Wissenschaft (1797), I, 2,

19: „Von jeher haben die alltäglichsten Menschen die größten Philosophen widerlegt, mit Dingen, die selbst
} 
und Mechanismus. Und gegen beide soll die Philosophie von Leibniz zentrale Argumente bereithalten. In Schellings Perspektive geht es um das Problem der Materie und die damit verbundene Frage nach ihrem Zusammenhang mit dem Geist, nach der Einheit von Unendlichem und Endlichem. „Der erste, der Geist und Materie mit vollem Bewußtseyn als Eines, Gedanke und Ausdehnung nur als Modifikationen desselben Princips ansah, war Spinoza. Sein System war der erste kühne Entwurf einer schöpferischen Einbildungskraft, der in der Idee des Unendlichen, rein als solchen, unmittelbar das Endliche begriff und dieses nur in jenem erkannte. Leibniz kam und ging den entgegengesetzten Weg (Schelling 1907, 20).“ Darum habe er, Leibniz, jenes „spekulative Hirngespinst einer Welt von Dingen an sich" aus seiner Theorie eliminiert. Die Vorstellungen äußerer Dinge sind nun Produkte eines inneren Prinzips. Eine äußere Einwirkung auf den Geist sei eine unmögliche, in sich widersprüchliche, nur scheinbar philosophische Auffassung, die am Wesen des Geistes, am Wesen der Perzeptionen vorbeigehe. Summa summarum: Leibniz „behauptete, daß sonach alle Veränderungen, aller Wechsel von Perceptionen und Vorstellungen in einem Geiste nur aus einem inneren Princip hervorgehen könne (Schelling 1907, 21).“ Natürlich ist diese Interpretation eingespannt in die nachkantische Diskussion um die Realität des Ding-an-sich, um die Realität eines Substrats der Außenwelt. Wie Fichte so bestritt auch Schelling entschieden, dass in der Kantischen Transzendentalphilosophie das Ding-an-sich mehr ist als ein bloßer Grenzbegriff. Ihm Realität zuzuschreiben hieße, die Kritische Philosophie Kants unter der Hand in einen Dogmatismus zurückzuverwandeln, der letztlich nichts anderes sei als der bekannte Dualismus, eine Philosophie, so Schelling wie Fichte, die nicht erkläre, was sie zu erklären vorgäbe. Als ein Protagonist auf dieser Bühne, ein Protagonist aus der Vorzeit, erscheint nun Leibniz, dessen Theorie, so Schellings Urteil über seine Zeitgenossen, heute nur noch zu den Kuriositäten gerechnet werde - zu Unrecht, wie er betont: „Heutzutage haben sich Leute zum Philosophiren gedrungen, die für alles andere, nur für Philosophie nicht, Sinn haben. Daher, wenn unter uns gesagt wird, daß keine Vorstellung in uns durch äußere Einwirkung entstehen könne, des Anstaunens kein Ende ist. Jetzt gilt es für Philosophie, zu glauben, daß die Monaden Fenster haben, durch welche die Dinge hinein und heraus steigen (Schelling 1907, 21).“

Im Hintergrund liegt die Auffassung, dass ein Leib-Seele Dualismus genau so wenig als Erklärungsmodell der Welt dienen könne wie der Dualismus von Materie und Geist. Kausalität, als Zusammenhang von Ursache und Wirkung, ist, so Schelling, nur im Bereich des Materiellen von Bedeutung. Die intelligible Welt ist dem Kausalnexus enthoben; sie ist das Reich der Freiheit und der Zwecke. Zerfällt die eine Welt, die als integrale Bestandteile sowohl kausaldeterminierte als auch freie Elemente oder Sphären enthält, damit nicht in zwei vollständig getrennte, unabhängige Welten? Ergibt sich nicht ein Weltdualismus ohne Vermittlungsinstanz? Ist der Geist völlig abgeschieden von der Materie, warum emanzipiert er sich nicht von ihr und lebt befreit von aller Erdenschwere in einem esoterischen Paradies, unbehelligt von den Hemmnissen und Widrigkeiten einer endlichen Materialität?

Kindern und Unmündigen begreiflich sind. (...) Kein Mensch denkt daran, dass sie vielleicht all das auch gewusst haben; denn wie hätten sie sonst gegen den Strom von Evidenz schwimmen können? Viele sind überzeugt, dass Plato, wenn er nur Locke lesen könnte, beschämt von dannen ginge; mancher glaubt, dass selbst Leibniz, wenn er von den Todten auferstünde, um eine Stunde lang bei ihm in die Schule zu gehen, bekehrt würde, und wie viele Unmündige haben nicht über Spinozas Grabhügel Triumphlieder angestimmt? - - “ 
Schelling lehnt eine solche Zwei-Welten-Theorie ab, - muss sie ablehnen, dies sowohl in Bezug auf die Philosophie Kants als auch auf diejenige Platons - und natürlich auch in Bezug auf Leibniz. Denn letztlich liefe diese Auffassung auf eine philosophische Theorie hinaus, die sich von der Welt abwendete, die deren Gehalte, insbesondere jene, die sich auf die Endlichkeit und Sinnlichkeit beziehen, vollständig aus der Philosophie eliminierte. In den Jahren vor der Jahrhundertwende indes interessiert sich Schelling brennend für jene Seite der Philosophie, der die Endlichkeit gerade eingeschrieben ist: die Natur. Sein Ziel war es zu zeigen, dass Endliches und Unendliches zugleich und ungetrennt sind. Dabei korrigiert er Spinoza, bei dem er zwar den Versuch findet, die Trennung des Realen und Idealen zu überwinden, allerdings jedoch einseitig, in einem objektiv aufgefassten Unendlichen, einem Unendlichen außer uns. „Man muß dieses System in sich selbst aufgenommen, sich selbst an die Stelle seiner [Spinozas, Ch. A.] Substanz gesetzt haben, um zu wissen, dass Unendliches und Endliches nicht außer uns, sondern in uns - nicht entstehen, sondern - ursprünglich, zugleich und ungetrennt da sind, und dass eben auf dieser ursprünglichen Vereinigung die Natur unseres Geistes und unser ganzes geistiges Daseyn beruht“" (Schelling 1907, 36f). ${ }^{6}$ Die Objektivität der Substanz des Spinoza müsse in eine Subjekt-Objektivität verwandelt werden, die substantiell und vorgängig bereits die Vereinigung von Endlichem und Unendlichem enthalte, allerdings nicht als solche, sondern als absolut vereinigte, eine Vereinigung, die neben Endlichem und Unendlichem auch Ideales und Reales, absolut Tätiges und absolut Leidendes eint. ${ }^{7}$ Aus einem äußeren absoluten Unendlichen heraus ließen sich zwar die Bestimmungen des Endlichen auffinden; die Trennung der beiden Sphären sei dann aufgehoben, aber nur dadurch, dass die andere unerklärlich faktisch, nicht aber durch einen „Übergang“ begründet sei. Einen Übergang vom Unendlichen zum Endlichen aber bewertet Schelling stets als einen Ungedanken.

In diesen Gedankengang kann Schelling die Monadenlehre unmittelbar integrieren. Leibniz habe einen entscheidenden Schritt getan, indem er seiner Philosophie den Begriff der Individualität zugrunde gelegt habe, ${ }^{8}$ womit Schelling sich auf den 9. Abschnitt der Monadologie bezieht, in dem Leibniz erklärt, dass es in der Natur niemals zwei vollkommen gleiche Wesen geben kann. Dabei verweist Schelling auf Jacobi: Dieser habe ,erwiesen, dass sein [Leibnizens; Ch. A.] System vom Begriff der Individualität ausgeht und darauf zurückkehrt. Im Begriff der Individualität allein ist ursprünglich vereinigt, was alle übrige Philosophie trennt, ...“(Schelling 1907, 37). Der Begriff der Individualität ist eine interpretierende Transformation des Begriffs der Monade. ${ }^{9}$ Sie

\footnotetext{
${ }^{6}$ Vgl. damit (Jacobi 1980, 261): "Folglich, wie ein Individuum gesetzt wird, so müssen nothwendig zugleich in ihm die Begriffe von Einheit und Vielheit, von Thun und Leiden, von Ausdehnung und Succession gesetzt werden; das heißt, es sind diese Begriffe jedem Individuo angebohrne oder anerschaffene Begriffe."

${ }^{7}$ Vgl.: (Schelling 1907, 37).

${ }^{8}$ Zum Gedanken der Individualität bei Leibniz vgl.: (Poser 2000,19-41, insb. 35-41).

9 Tatsächlich läßt sich dieser Gedanke, der Gedanke nämlich, daß das Individuum nichts anderes ist als die Einfaltung und Einschränkung des Alls oder Gottes selbst, bereits in allgemeiner Formulierung aus Anaxagoras, Fr. 6, herauslesen. Neben Albert, Thomas und Eckhart findet sich ein Anschluß an Anaxagoras vor allem bei Nikolaus von Kues: "In qualibet enim creatura universum est ipsa creatura, et ita quodlibet recipit omnia, ut in ipso sint ipsum contracte. Cum quodlibet non possit esse actu omnia, cum sit contractum, contrahit omnia, ut sint ipsum." De docta ignorantia, II, cap. V, n. 117. Vgl. Dazu: (Flasch 1998, 101, 146f); ferner: (Rang 2000, 103-110). - Für Flasch kündigt sich hier eine "individualisierte Konzeption der Wahrheit" an, welche die Welt als ganze im Individuum auf individualisierte Weise spiegelt. Es gebe, so Flasch, bei Cusanus kein "objektives" Drittes mehr, in dem sich die Wahrheiten ausgleichen ließen. Indes betont Cusanus, daß zugleich für jedes
} 
enthalte, so Schelling, Unendliches und Endliches als ursprünglich Vereinigte. Der Übergang vom Unendlichen zum Endlichen „findet sich nur da nicht, wo Endliches und Unendliches ursprünglich vereinigt sind, und diese ursprüngliche Vereinigung ist nirgends, als im Wesen einer individuellen Natur. Leibniz ging also weder vom Unendlichen zum Endlichen, noch von diesem zu jenem über, sondern beides war ihm auf einmal - gleichsam durch eine und dieselbe Entwicklung unserer Natur - durch eine und dieselbe Handlungsweise des Geistes wirklich gemacht" (Schelling 1907, 37).

Diese vorgängige Einheit von Unendlichem und Endlichem findet sich in den Vorstellungen. Diese seien bei Leibniz zugleich endlich und unendlich. Endlich nämlich zeigen sich die Vorstellungen in ihrer Aufeinanderfolge, unendlich aber durch die Endlosigkeit dieser Sukzession. Die Vorstellung hat daher an sich selbst eine doppelte Natur, die sie aus der Subjektivität ererbt: Einerseits ist sie singulärer und endlicher Punkt einer Kette von Vorstellungen, daher begrenzt und beschränkt, andererseits aber Glied einer solchen Kette von Vorstellungen, die endlos, daher unendlich ist.

Diese Sukzession habe bei Leibniz den Charakter der Notwendigkeit. Die Dinge entstünden nämlich in uns gleichzeitig mit den Vorstellungen, durch unsere Natur, durch ein inneres Prinzip in uns, in unserer eigenen Individualität. Das Individuum, die Monade, wird daher zur Projektionsfläche für die Welt, die allerdings keine von der Projektion selbst unabhängige Selbständigkeit besitzt, sondern sich in der Projektion erschöpft. In diesem Sinne sind Ding und Vorstellung untrennbar, oder, wie Schelling sich ausdrückt, gleichzeitig. Es gibt weder eine Vorrangigkeit des Dings im Erkenntnisprozess noch eine des vorstellenden Subjekts, sondern nur die absolute vorgängige Gleichrangigkeit, bzw. Identität beider.

Die Frage nach dem ursprünglich Realen im Erkenntnisprozess kann hier nur so beantwortet werden, dass es sich dabei um das vorstellende Wesen selbst handeln müsse. Dieses sei im eminenten Sinne wirklich, alles endliche Wirkliche, sei wirklich durch es und vermittelst seiner. Das vorstellende Wesen - als die vorgängige Einheit - entwickelt aus sich heraus alles, was im abgeleiteten Sinne wirklich ist. Hier schleicht sich in Schellings Ausführungen eine transzendentalphilosophische Überlegung ein, die ganz an das Programm Fichtes erinnert: Alle Realität ist im Ich gesetzt und alles, was als real erscheint, hat die Realität übertragen bekommen aus dem Ich: "Aller Realität Quelle", so Fichte, "ist das Ich"(Fichte 1962, 293). Äußere Dinge werden wirklich durch die Art und Weise, in der ein geistiges Wesen vorstellt, denn nur ein solches Wesen ist etwas, "das in sich selbst Quell und Ursprung seines Daseyns trägt" (Schelling 1907, 38), so Schelling. Damit aber sind die Vorstellungen mehr als bloße Blasen, die im Subjekt aufsteigen. Die erzeugende Kraft der Vorstellungen ist nun das transzendentale Subjekt selbst.

Hier setzt Schellings Interpretation der prästabilierten Harmonie an, und tatsächlich hält Schelling die prästabilierte Harmonie für einer Interpretation bedürftig. Sein

existierende Ding nicht nur alles in ihm es selbst, sondern es selbst in Gott Gott sei. Cusanus feiert die bewundernswürdige Einheit, die staunenswerte Gleichheit und bewunderungswürdigste Verbindung der Dinge, so daß alles in allem sei. ("Mirabilem rerum unitatem, admirandam aequalitatem et mirabilissimam vides conexionem, ut omnia sint in omnibus" De docta ignorantia, II, cap. V, n. 120) Die Trinität (Einheit, Gleichheit und Verbindung; vgl. De docta ignorantia I, cap. VII) ist die Struktur der Welt sowie des Individuums, seiner Ein- und Ausfaltung, letztlich die Struktur Gottes. Auch für Leibniz ergibt sich trotz des monadologischen Wahrheitsbegriffs ein "bewundernswerter Einklang der Phänomene der unterschiedlichen Substanzen" (vgl. Poser 2000, 37). Die Perspektivierung des Wahrheitsbegriffs - dies gilt für Cusanus wie für Leibniz - zielt auf die Universalisierung der Wahrheit, nicht auf deren bloße Individualisierung. 
Unbehagen bezieht sich auf die Willkürlichkeit einer vorgängigen Übereinstimmung: nicht zwischen den einzelnen vorstellenden Substanzen und den vorgestellten Gegenständen, was einer monadischen Theorie widerspräche, sondern einer Übereinstimmung zwischen den vorstellenden Individuen selbst. Damit berührt Schelling ein Zentralproblem der transzendentalen Erkenntnistheorie: die Frage nach dem transzendentalen Subjekt: Es ist weder restringiert auf die Bewusstseinsakte einer singulären Person noch - abstrahiert von allen konkreten Bewusstseinsakten - als eine Vernunft für alle vernünftigen Wesen, noch ist es eine Vernunft in allen Vernunftakten, noch ist es eine von Gott gestiftet identische Ausstattung aller Individuen, die sie alles Gleiche auf gleiche Weise und als Gleiches erkennen lässt. ${ }^{10}$ In allen diesen Versuchen, die Vielheit der Bewusstseinsakte, die einer Vielheit von Individuen zugehören, zu einer Welt zu synthetisieren, entdeckt Schelling den Mangel, dass die Erklärung bei einer bloßen Faktizität anhebt. "Diese Uebereinstimmung unserer Natur aber durch eine prästabilierte Harmonie erklären, heißt sie wirklich nicht erklären. Denn dieses Wort sagt nur, daß eine solche Uebereinstimmung stattfinde, aber nicht wie und warum" (Schelling 1907, 38). Außerdem würde man - nach Schelling - etwas bemühen, das zuvor als Grund der Vorstellungen ausgeschlossen wurde, nämlich eine Kausalität der Außenwelt, in diesem Falle eine dem Individuum gegenüber vorrangige äußere vernünftige Synchronisierung aller immanenten Akte: Das Individuum wäre nicht länger Selbstgrund seines Wissens und Erkennens. Umgekehrt: Aus der Immanenz der individuellen Substanz muss- als das Wesen der endlichen Natur - die Übereinstimmung der Vorstellungen folgen. Erst dann wäre die Welt in ihrer Existenz und ihrer Ordnung für das vorstellende Individuum nicht bloß zufällig. Die Intelligibilität gehört nicht zur Dingwelt, in der Entstehen und Vergehen durch äußere Kausalmechanismen erklärt werden könnten. Nach Schelling habe Leibniz behauptet, ein geistiges Wesen könne überhaupt nicht entstanden sein, sondern sei der Kausalität vollständig enthoben. Damit bezieht sich Schelling auf die grundlegenden Abschnitte (4 und 5) der Monadologie, in denen Leibniz argumentiert, dass die Monade, weil sie einfache Substanz sei, weder auf natülichem Wege entstehen noch vergehen könne. Allerdings zieht Leibniz einen Akt der Schöpfung in Betracht (Abschnitt 6), d. h. eine göttliche Kausalität die zur Entstehung einer Monade geführt habe. Schelling hingegen betont in seiner Interpretation, die Monade bei Leibniz sei "absoluter Selbstgrund" ihres Seins und Wissens, ${ }^{11}$ causa sui, damit auch Schöpferin ihrer selbst oder besser: unerschaffene und unerschaffbare Autonomie. Damit ist die Stelle eines wirkenden Gottes, wie Leibniz sie in der Monadologie besetzte, bei Schelling vakant geworden, ein Gott, der bei Leibniz noch Garant war für die ewigen Wahrheiten, ein Gott, dessen Wahl des Besten das Prinzip ist auch der zufälligen Wahrheiten (Abschnitt 46).

Nach Schelling ist die Monade das, was sie ist, dadurch, dass sie ist: Ihr Sein bestimmt ihr Wesen, "ein Wesen, zu dessen Natur auch dieses bestimmte System von Vorstellungen äußerer Dinge gehört" (Schelling 1907, 39). Darum sei Philosophie nach Leibniz zunächst nichts anderes als "Naturlehre unseres Geistes". Aber diese Natur ist dem Geist immanent, keine äußere Natur, die etwa unter dem Gesetz der Kausalität stehen müsste. Nach Schelling erreicht die Philosophie mit dieser Einsicht eine neue Stufe: Sie überwindet den Dogmatismus durch die genetische Potenz einer transzendentalen Einheitslehre. Die Vorstellungen, die Gesetze, die ihnen eine systematische Form geben, der

\footnotetext{
${ }^{10}$ Vgl.: (Schelling 1907, 39)

${ }^{11}$ Vgl.: (Schelling 1907, 39).
} 
ganze Gehalt unseres Bewusstseins lässt sich nun genetisch aus der Immanenz entwickeln. "Von nun an", so Schelling enthusiastisch, "ist zwischen Erfahrung und Spekulation keine Trennung mehr" (Schelling 1907, 39). Die Welt der Empirie ist mit der der apriorischen Begriffe versöhnt, dies nicht etwa in einem nachträglichen Akt konkreter Erkenntnis, wie dies bei Kant gedacht wurde, sondern vor aller Erkenntnis als ihre erste Möglichkeitsbedingung: Natur und Geist in unmittelbarer ursprünglicher Einheit, getrennt - wie es die Einleitung zu den Ideen zu einer Philosophie der Natur in Anspielung auf mythologische Erzählformen präsentiert - durch das Wirken der trennende Reflexion, um durch die Philosophie in Freiheit zurückzukehren in die ursprüngliche Einheit.

Der Begriff der Individualität erscheint hier durch die Rezeption der Leibnizschen Monadologie geprägt zu sein. Und so dominiert dieser Begriff die Abhandlungen Schellings zur Naturphilosophie, insbesondere dann, wenn es ihm um den Organismus geht, das organische Ganze, das bei Schelling als Leitbegriff der Naturphilosophie im Gegensatz zum Mechanismus gelten dürfte. ${ }^{12}$

In der Identitätsphilosophie, als deren spätes Zeugnis hier der Dialog Bruno angeführt werden soll, ist die Monadenlehre, für die in der Naturphilosophie noch Leibniz stehen durfte, ganz in die eigene Theorie transformiert. Es ist nicht mehr zu erkennen, dass dieser Baustein einmal einem anderen Theoriebezug zugehörte. Jetzt spricht Schelling aus der Perspektive einer platonisch gewendeten Ideen- und Einheitstheorie. Das Grundproblem liegt nicht mehr im Horizont einer transzendentalen Immanenztheorie, sondern - im Horizont einer Ideenlehre - auf der Vermittlung der ewigen Idee mit dem sinnlichen Ideat. Als Paradigma dieses Verhältnisses dient Schelling die Idee der Schönheit; denn in wohl keinem Bezug einer Idee zu ihrem sinnlichen Abbild wird deutlicher, dass das Sinnliche aufgrund seiner Sinnlichkeit individuell und schön ist: Die Einzigartigkeit des Kunstwerks, die Singularität des Schönen, ist nachgerade das Signum der Idee. Damit ist aber zugleich die Frage nach der Individualität gestellt.

Als Grund der Individualisierung ist die Idee der Schönheit ausgeschlossen. Sie - als ewige und intelligible Entität hat - mit dem Sinnlichen und Endlichen nichts gemein. Als Vermittlungsstufe erscheint bei Schelling zunächst das hervorbringende Individuum, damit zugleich ein ganz neues - und wie es scheint - ganz modernes Problem: das Problem der Individualität von Künstler und Kunstwerk. Man kann diese Fragestellung aber durchaus als exemplarisch ansehen für das Problem des Einzeldings überhaupt.

Die Formel für die Verbindung des Endlichen, Unendlichen und Ewigen lautet: Durch das Ewige ist das Endliche mit dem Unendlichen verknüpft. Daher muss Schelling auch das Problem der Individualität durch die Ideenlehre lösen: "Das Ewige demnach bezieht sich auf alle Dinge durch ihre ewigen Begriffe, auf das hervorbringende Individuum also durch den ewigen Begriff des Individuums, der in Gott und mit der Seele ebenso eins ist wie die Seele mit dem Leibe. (...) Diesen ewigen Begriff des Individuums also werden wir als das Hervorbringende eines Werkes ansehen, worin die höchste Schönheit dargestellt ist" (Schelling 1907 (2), 123). Der ewige Begriff des Individuums ist nach zwei Seiten hin durch Einheit gekennzeichnet; er ist einerseits in Gott eins, aber andererseits genau so eins mit der Seele. Die Seele wiederum ist eins mit dem Leib.

Transzendentaler Grund der Existenz des Individuellen ist die ewige Idee des Individuums - ein Baustein, genommen aus der Rezeption der Monadologie, der nun die

${ }^{12}$ Vgl. : (Schelling 1907 (I), 518ff). 
Verbindung zwischen Ideenwelt und Sinnenwelt stiften soll. Tatsächlich benötigt Schellings Theorie ein solches Verbindungsstück - dies gerade in Bezug auf die Kunst. Hier tritt das singuläre Werk in seiner Singularität in den Brennpunkt des philosophischen Interesses. Wenn alle Dinge ihr wesentliches Sein in ihrem ewigen Begriff haben, so muss dies auch für das hervorbringende Individuum, also das konkrete künstlerische Genie gelten. Daraus folgt aber: Das konkrete Individuum ist seinem wesentlichen Sein nach gar nicht dieses Individuum, sondern die Idee des Individuums, ewiger Begriff des Individuums. Das anvisierte konkrete künstlerische Individuum hat sich also unter der Hand entindividualisiert. Die Frage nach der künstlerischen Hervorbringung, die sich für Schelling gerade an der Singularität des Schönen entzündete, ist in den Bereich des Absoluten zurückgenommen.

Die Interpretation der Leibnizschen Monadenlehre ist bei Schelling transformiert in den eigenen Entwurf einer Identitätstheorie. Dies scheint ein der Philosophie selbst eingeschriebenes Verfahren zu sein: Theoriestücke vergangener Positionen werden $\mathrm{zu}$ Bausteinen neuerlicher Theoriebildungen, werden verwandelt und erlangen in verwandelter Gestalt neue Bedeutung. Zu denken geben muss nur die Tatsache, dass dieser Transformation des Vergangenen oft nur eine unzureichende, bisweilen einseitige, oft kurzsichtige, in jedem Fall aber eine ahistorische Interpretation zugrunde liegt. So interpretiert Schelling in der Tat die Monadenlehre, aber er interpretiert sie von vornherein im Bezugsrahmen der nachkantischen Transzendentalphilosophie: im Horizont der Frage nach dem Ding an sich, der Frage nach der Realität der Außenwelt, der Frage nach der Bewusstseinsimmanenz alles Seienden. Dadurch erscheint die Konzeption von Leibniz insgesamt verkürzt: Die Funktion Gottes als Schöpfer, als Garant einer prästabilierten Harmonie und Stabilisator der Vernunftwahrheiten, als ursprüngliche einfache Substanz (Abschnitt 47) muss in der Transzendentalphilosophie Schellingscher Ausprägung gestrichen werden. Für sie ist kein Ort mehr vorgesehen. An ihre Stelle rückt eine Subjekt-Objekt-Konzeption, in der die Individualität zwar abstrakt eingelassen ist, deren Basis aber eine Theorie der Selbstverursächlichung bildet, neben der und außerhalb ihrer ein philosophischer Gott nicht bestehen kann. Außerdem fehlen bei Schelling - das sei hier nur grob angedeutet wesentliche Aspekte: etwa die politischen, sozialen und ethischen.

Andererseits verdichten sich bei Schelling die sachlichen Probleme. Was bedeutet es für eine Identitätstheorie, dass sie an einer zentralen Stelle von jenem Begriff konstitutiven Gebrauch macht, der gerade auf der Unterschiedenheit beruht? Dass etwas von allem anderen unterschieden, nämlich individuell ist, soll zugleich dem zugehören, was das wesentliche Sein von allem ausmacht, d. h. in allem identisch: ununterschieden ist. Das von allem Unterschiedene müsste zugleich das Ununterschiedene sein. Erst eine solche Theorie, die Unterschiedenheit und Ununterschiedenheit miteinander vermittelt denken könnte, würde Schellings Problem lösen, wie der Bereich der ewigen Idee $\mathrm{zu}$ vermitteln ist mit den endlichen, singulären Ideaten. Hierzu wäre aber neben, nicht unterhalb der Identität auch Nicht-Identität in der ewigen Idee anzusetzen - eine Konsequenz, die Schelling 1802 noch nicht zu ziehen bereit war. 


\section{VERWEISE}

Fichte, J.G. Grundlage der Gesamten Wissenschaftslehre, in J.G.Fichte-Gesamtausgabe der Bayerischen Akademie der Wissenschaft (=GA), Bd.I. (Hg.) Lauth, Reinhard - Jacob, Hans - Gliwitzky, Hans. Stuttgard-Bad Cannstatt, 1962.

Flasch, Kurt. Nikolaus von Kues. Geschichte einer Entwicklung. Frankfurt a. M.: 1998.

Hartmut, Hecht. Die Zeit, Leibniz' zu verstehen, ist gekommen', in: Vorträge zur Philosophie Schellings. Berliner Schelling Studien, H. 1, Berlin, 2000.

Hegel, G.W.F. Wissenschaft der Logik, I, in Gesammelte Werke (11), Hamburg: Felix Meiner Verlag, 1978.

Jacobi, F.H. David Hume über den Glauben, oder Idealismus und Realismus. In Friedrich Heinrich Jacobi's Werke. (Hg.) Roth, Friedrich - Köppen Friedrich. Bd 2, Leipzig 1815. Repr. Nachdr.Darmstadt, 1980.

Poser, Hans. Phaenomenon bene fundamentum. Leibnizens Monadologie als Phänomenologie, in: Phänomenologie und Leibniz. (Hg.) Cristin, Renato - Sakai, Kiyoshi, 35-41, insb. 19-41. Freiburg/München: 2000.

Rang, Bernhard. Identität und Indifferenz. Eine Untersuchung zu Schellings Identitätsphilosophie. Frankfurt a. M.: 2000 .

Kaehler, Klaus Erich, Von der monadischen zur transzedentalen Subjektivität. Die Bedingtheit in der Unbedingtheit des Subjekts bei Leibniz und Kant. In Philosophie der Subjektivität? Zur Bestimmung des neuzeitlichen Philosophierens. Akten des 1. Kongresses der Internationalen Schelling-Gesellschaft 1989. (Hg.) Baumgartner, Hans Michael - Jacobs, Wilhelm G. Stuttgart Bad-Cannstatt, 1993.

Schelling, F.W.J. Ideen zu einer Philosophie der Natur als Einleitung in das Studium dieser Wissenschaft (1797), I, 2. Leipzig: Fritz Eckardt, 1907.

Schelling, F.W.J. Von der Weltseele. (1798), I, 2. Leipzig: Fritz Eckardt, 1907.

Schelling, F.W.J. Bruno. (1802), I, 4. Leipzig: Fritz Eckardt, 1907.

\section{LAJBNIC, I PITANJE IDENTITETA I INDIVIDUALITETA U MIŠLJENJU F. V. J. ŠELINGA}

Šelingovo tumačenje Lajbnicove teorije o mondama transformisano je u njegovom sopstvenom projektu teorije identiteta. Čini se da je to postupak koji čini sastavni deo filozofije - delovi teorija ranijih filozofskih pozicija postaju građa za izgradnju novih teorija, transformišu se i zadobijaju novo značenje u transformisanom obliku. Ono o čemu treba razmišljati je činjenica da je ovaj preobražaj onog prošlog često utemeljen na neadekvatnom, ponekad jednostanom, često kratkovidom, ali u svakom slučaju anahronom tumačenju. Šeling tako zaista interpretira teoriju monada, ali on je od samog početka tumači u okviru postkantovske transcedentalne filozofije, $u$ horizontu pitanja stvari po sebi, pitanja o postojanju spoljašnjeg sveta, pitanja o imanenciji svesti onog bivstvujućeg. Posledica toga je skraćivanje Lajbnicove ukupne filozofije. Funkcija boga kao stvoritelja, garanta prestabilirane harmonije i stabilizatora umstvenih istina, odnosno, izvorne jednostavne substance, u Šelingovoj transcendentalnoj filozofiji mora se izbrisati. Za nju nema mesta. Na njeno mesto dolazi koncepcija subjekt-objekt, u kojoj se individualnost predstavlja na apstraktan način, čija je osnova teorija o samouzročnosti, u kojoj ili izvan koje ne može postojati filozofski bog. Osim toga kod Šelinga takođe nedostaju politički, društveni i etički aspekti.

Ključne reči: Lajbnic, Šeling, interpretacija, učenje o monadama, identitet, individualitet. 